\title{
Use of an intra-aortic balloon pump during laparoscopic sleeve gastrectomy
}

\begin{abstract}
Andres Narvaez, Juan Esteban Perez, Melissa Castro, Keri A. Seymour
Department of Surgery, Duke University Health System, Division of Metabolic and Weight Loss Surgery, Durham, NC 27704, USA
\end{abstract}

\begin{abstract}
Correspondence to: Assistant Prof. Keri A. Seymour, DO, FACS, Division of Metabolic and Weight Loss Surgery, Duke University, 407 Crutchfield Street, Durham, NC 27704, USA. E-mail: keri.seymour@duke.edu
\end{abstract}

How to cite this article: Narvaez A, Perez JE, Castro M, Seymour KA. Use of an intra-aortic balloon pump during laparoscopic sleeve gastrectomy. Mini-invasive Surg 2020;4:31. http://dx.doi.org/10.20517/2574-1225.2020.22

Received: 15 Feb 2020 First Decision: 30 Mar 2020 Revised: 13 Apr 2020 Accepted: 26 Apr 2020 Published: 16 May 2020

Science Editor: Wah Yang Copy Editor: Jing-Wen Zhang Production Editor: Tian Zhang

\begin{abstract}
Heart transplant is the primary treatment for end-stage heart failure; however, morbid obesity limits candidacy. Bariatric surgery performed in patients with advanced heart failure improves eligibility for heart transplantation. This is the first report of an intra-aortic balloon pump used during laparoscopic sleeve gastrectomy. A patient with morbid obesity and non-ischemic cardiomyopathy was referred for weight loss surgery prior to evaluation for heart transplantation. An intra-aortic balloon pump was placed for aggressive diuresis and cardiovascular support during laparoscopic sleeve gastrectomy. The patient did not suffer any complications or require readmission. The use of an intra-aortic balloon pump as a mechanical circulatory system provided a safe laparoscopic sleeve gastrectomy in a patient with advanced heart failure.
\end{abstract}

Keywords: Heart failure, sleeve gastrectomy, bariatric surgery, cardiac transplant

\section{INTRODUCTION}

Heart failure (HF) affects approximately 5.7 million adults in the United States with 5 -year mortality approaching $50 \%{ }^{[1]}$. Heart transplantation is the standard management for advanced HF. However, a body mass index (BMI) $\geq 35 \mathrm{~kg} / \mathrm{m}^{2}$ is associated with increased early complications, decreased longterm survival, and lower likelihood of receiving an organ ${ }^{[2,3]}$. Weight loss results in decreased vascular stiffness and reduction in ventricular hypertrophy ${ }^{[4]}$. Bariatric surgery in patients with advanced HF may result in eligibility for heart transplantation. We report the use of an intra-aortic balloon pump (IABP) for perioperative cardiovascular support during laparoscopic sleeve gastrectomy (LSG) in a patient with advanced HF. 


\section{CASE REPORT}

A 43-year-old patient with non-ischemic cardiomyopathy, New York Heart Association (NYHA) Class IIIb, and morbid obesity (BMI $45 \mathrm{~kg} / \mathrm{m}^{2}$ ) was referred for weight loss prior to evaluation for heart transplantation. Co-morbid conditions included obstructive sleep apnea, nonalcoholic steatohepatitis, gastroesophageal reflux disease, hyperlipidemia, exertional hypotension, adrenal insufficiency, and $3 \mathrm{~L}$ continuous oxygen. The patient was diagnosed with HF three years prior with an ejection fraction (EF) of 15\%-20\%, which required an implantable cardioverter-defibrillator (ICD). Titration of antihypertensive and diuretic medications was limited by syncope and hypotension. The patient was hospitalized over thirteen times with episodes of HF exacerbation and arrhythmias. Ultimately, the ICD was converted to a biventricular device, and EF improved to $25 \%$ with complete ventricular pacing. Our patient did not qualify for left ventricular assist devices (LVAD), but still, weight loss surgery was recommended to improve cardiac function and increase the potential for candidacy for heart transplant.

\section{Preoperative care}

Our multidisciplinary team consisted of advanced HF and transplant cardiology, cardiothoracic surgery, cardiothoracic anesthesiology, and bariatric surgery. Diet modifications resulted in successful weight loss of 9 pounds over ten months. Preoperative testing included an upper gastrointestinal series that revealed normal esophageal motility, no hiatal hernia, and no gastroesophageal reflux. LSG was chosen over Rouxen-Y gastric bypass for technical ease, shorter operative time, perioperative safety profile, and effective weight loss in end-stage HF patients ${ }^{[5-7]}$.

To address the perioperative risks of volume shifts and hemodynamic instability, preoperative placement of an IABP was considered. The IABP was necessary to establish euvolemia prior to surgery and maintain adequate cardiac output during laparoscopy. Over six months, the patient obtained clearances from nutrition, psychology, endocrinology, pulmonology, and cardiology. The patient was pre-admitted to the advanced HF team to address fluid shifts prior to surgery. An IABP was placed on hospital day 2 to prevent hypotension with ongoing, supervised diuresis. A catheter was inserted through the right femoral artery with a $7.5 \mathrm{~F}$ sheath and advanced under fluoroscopic guidance into the descending thoracic aorta. The IABP was turned on with continuous heparin infusion until 6 hours prior to surgery. Successful diuresis was achieved with a negative fluid balance of 5 liters, without episodes of hypotension.

\section{Surgical technique}

Upon arrival to the operating room, the IABP was transferred from battery to an alternating current power. After additional arterial and venous access was achieved, the case began with the placement of four ports in the subcostal area with the option of low-pressure insufflation. The patient was gradually positioned in reverse Trendelenburg as hemodynamic status was monitored. A liver retractor was placed to expose the gastroesophageal junction. The greater curvature of the stomach was mobilized to the left crus with cauterization of the short gastric blood vessels. Hemostasis was achieved and a $36 \mathrm{~F}$ bougie was passed into the gastric lumen. Sleeve gastrectomy was performed using staplers, at 4-5 $\mathrm{mm}$ and 3-4 mm staple heights, with staple line reinforcement. Care was taken to ensure the incisura was not narrowed. An esophagogastroduodenoscopy was performed with a negative leak test and symmetric stomach. Blood loss was less than $50 \mathrm{~mL}$ and hemodynamic stability persisted throughout the case. The patient tolerated the procedure without any complications and the continuous heparin infusion was restarted.

\section{Post-operative outcomes}

Diet was advanced on post-operative day (POD) 1 and the IABP was removed on POD 2. The patient was discharged on POD 9 without complications, blood transfusions, or readmission. Follow-up visits with the bariatric surgeon, dietitian, psychologist, or advanced HF cardiologist occurred at 3 weeks, 3 months, 6 months, and 1 year. Progressive weight loss occurred at 3 weeks with 8 percent excess weight loss (\% 
EWL), 3 months with $21 \%$ EWL, and 6 months with 27\% EWL. At 12 months, the patient had 39\% EWL, reached a BMI of $36 \mathrm{~kg} / \mathrm{m}^{2}$, and was 11 pounds from the goal weight. Exercise tolerance improved, oxygen was no longer required, and NYHA Class II-III symptoms were noted. In addition, hospitalizations for HF decreased to two admissions over one year and EF was stable at $25 \%$. By 15 months, the patient presented in cardiogenic shock and ultimately required LVAD placement for cardiac stabilization.

\section{DISCUSSION}

Obesity is a risk factor for the development of cardiovascular disease and HF. Heart transplant is the primary treatment for end-stage HF; however, morbid obesity is a relative contraindication to transplantation ${ }^{[8]}$. A BMI $\geq 35 \mathrm{~kg} / \mathrm{m}^{2}$ is associated with early complications and decreased long-term survival after heart transplant, compared to class I obesity ${ }^{[2]}$. Weight loss improves cardiovascular function through increased left ventricular diastolic and systolic function, reduction of myocardial oxygen consumption, and reversal of impaired aortic distensibility ${ }^{[4]}$. The Swedish Obese Subjects study demonstrated sustained weight loss and decreased cardiovascular events, including death, after bariatric surgery $^{[9]}$. Thus, bariatric surgery is an effective intervention for morbidly obese patients who require weight reduction to become a candidate for heart transplantation.

Bariatric surgery in patients with severe cardiomyopathy, including LVAD, is an opportunity for rapid weight loss as a "bridge" to transplantation. Studies of small cohorts show patients with LVAD who underwent bariatric surgery had improvement in median left ventricular ejection fraction and reduction in NYHA classification ${ }^{[6,10]}$. In particular, Punchai et al. ${ }^{[6]}$ reported on three patients with LVAD who went on to receive a heart transplant after LSG. Acceptable rates of complications included five perioperative morbidities and two deaths from LVAD complications. LVAD complications occur at a rate of $8 \%-29 \%$ and include: bleeding, infection, neurologic event, and anticoagulation issues ${ }^{[11]}$. In addition, mechanical circulatory support devices are associated with heightened healthcare costs. The total cost for LVAD as a bridge to transplantation ranges from $\$ 316,078$ to $\$ 1,025,500^{[12]}$. Further cost of management includes the median cost of a single readmission at $\$ 7,546$; with up to $81.8 \%$ of LVAD patients requiring readmission ${ }^{[12]}$. Options for our patient were to perform weight loss surgery: (1) after further deterioration that required LVAD implementation or (2) with the option of temporary mechanical support with IABP. The early intervention of LSG improved cardiac function and symptoms for more than one year although, an episode of cardiogenic shock ultimately required LVAD placement. While the progression to LVAD is an acceptable risk, this case describes the multidisciplinary team approach critical to successful LSG in a patient with complex heart disease.

To the best of our knowledge, this is the first report of the temporary use of an IABP to achieve uncomplicated bariatric surgery in a high-risk patient with advanced cardiac disease. The IABP has been utilized to establish hemodynamic stability in patients awaiting cardiac surgery with decompensated $\mathrm{HF}^{[13]}$, but rarely reported in non-cardiac surgery. Successful placement of an IABP in patients with congestive HF who underwent non-cardiac procedures (nephrectomy, colectomy with splenectomy, and an exploratory laparotomy) potentially reduced morbidity and mortality ${ }^{[14]}$. Similarly, the temporary mechanical circulatory assistance of the IABP resulted in a successful LSG, without morbidity or mortality. As a result, the multidisciplinary team approach resulted in safe bariatric surgery, in a hospital with the infrastructure to manage advanced HF.

In conclusion, the temporary application of an IABP provides cardiovascular support to achieve a safe LSG. A multidisciplinary team approach is recommended for perioperative management of advanced HF. 


\section{DECLARATIONS}

\section{Authors' contributions}

Made substantial contributions to the conception, writing, and literature review of the case report: Narvaez A, Perez JE, Castro M, Seymour KA

\section{Availability of data and materials}

Not applicable.

\section{Financial support and sponsorship}

None.

\section{Conflicts of interest}

All authors declared that there are no conflicts of interest.

\section{Ethical approval and consent to participate}

Not applicable.

\section{Consent for publication}

Not applicable.

\section{Copyright}

(c) The Author(s) 2020.

\section{REFERENCES}

1. Mozaffarian D, Benjamin EJ, Go AS, Arnett DK, Blaha MJ, et al.; American Heart Association Statistics Committee; Stroke Statistics Subcommittee. Heart disease and stroke statistics-2016 update: a report from the american heart association. Circulation 2016;133:e38360 .

2. Nagendran J, Moore MD, Norris CM, Khani-Hanjani A, Graham MM, et al. The varying effects of obesity and morbid obesity on outcomes following cardiac transplantation. Int J Obes (Lond) 2016;40:721-4.

3. Weiss ES, Allen JG, Russell SD, Shah AS, Conte JV. Impact of recipient body mass index on organ allocation and mortality in orthotopic heart transplantation. J Heart Lung Transplant 2009;28:1150-7.

4. Rider OJ, Francis JM, Ali MK, Petersen SE, Robinson M, et al. Beneficial cardiovascular effects of bariatric surgical and dietary weight loss in obesity. J Am Coll Cardiol 2009;54:718-26.

5. Hawkins RB, Go K, Raymond SL, Ayzengart A, Friedman J. Laparoscopic sleeve gastrectomy in patients with heart failure and left ventricular assist devices as a bridge to transplant. Surg Obes Relat Dis 2018;14:1269-73.

6. Punchai S, Nor Hanipah Z, Sharma G, Aminian A, Steckner K, et al. Laparoscopic sleeve gastrectomy in heart failure patients with left ventricular assist device. Obes Surg 2019;29:1122-9.

7. Chaudhry UI, Kanji A, Sai-Sudhakar CB, Higgins RS, Needleman BJ. Laparoscopic sleeve gastrectomy in morbidly obese patients with end-stage heart failure and left ventricular assist device: medium-term results. Surg Obes Relat Dis 2015;11:88-93.

8. Mancini D, Lietz K. Selection of cardiac transplantation candidates in 2010. Circulation 2010;122:173-83.

9. Sjöström L, Peltonen M, Jacobson P, Sjöström CD, Karason K, et al. Bariatric surgery and long-term cardiovascular events. JAMA 2012;307:56-65.

10. McCloskey CA, Ramani GV, Mathier MA, Schauer PR, Eid GM, et al. Bariatric surgery improves cardiac function in morbidly obese patients with severe cardiomyopathy. Surg Obes Relat Dis 2007;3:503-7.

11. Kadakia S, Moore R, Ambur V, Toyoda Y. Current status of the implantable LVAD. Gen Thorac Cardiovasc Surg 2016;64:501-8.

12. Seco M, Zhao DF, Byrom MJ, Wilson MK, Vallely MP, et al. Long-term prognosis and cost-effectiveness of left ventricular assist device as bridge to transplantation: a systematic review. Int J Cardiol 2017;235:22-32.

13. Naqvi SY, Salama IG, Yoruk A, Chen L. Ambulatory intra aortic balloon pump in advanced heart failure. Card Fail Rev 2018;4:43-5.

14. Foster ED, Olsson CA, Rutenburg AM, Berger RL. Mechanical circulatory assistance with intra-aortic balloon counterpulsation for major abdominal surgery. Ann Surg 1976;183:73-6. 